\title{
Guest Editors' Introduction to Special Issue on the Third INFORMS Simulation Society Research Workshop
}

The 2011 INFORMS Simulation Society Research Workshop was held in Montreal during the period of July 18-20, 2011. The workshop was sponsored and organized by the GERAD research center and also sponsored by the National Science Foundation (USA) under Grant Number CMMI-1132167, and by IBM. The general goal of the INFORMS Simulation Society workshops is to bring together leading researchers working in simulation methodology and applications related to operation research and management science, to reflect on a selected central theme and identify important issues that need attention and promising paths to solutions.

The theme of the 2011 workshop was simulation in the management of complex service systems, such as healthcare systems, emergency systems, call centers, logistic and delivery systems, transportation systems, telecommunication networks, revenue management systems, and smart energy grids. Questions of interest included (among others) defining appropriate stochastic models for these systems, constructing effective tools to optimize or improve decision making via simulation, transferring ideas and methods across application areas, and identifying the main important challenges for a research agenda in these areas.

The workshop featured ten 1-hour invited plenary talks, complemented by 34 poster presentations. The plenary talks presented were:

John Fowler, Arizona State University, "Simulation of Healthcare Systems"

Peter Glynn, Stanford University, "Traffic Modeling for Complex Service Systems"

David Goldsman, Georgia Institute of Technology, "Modeling and Simulation of Pandemic Influenza Outbreaks"

Peter J. Haas, IBM Almaden Research Center, San Jose, "Composite Simulation Modeling of Complex Service Systems: Example and Research Challenges"

Shane G. Henderson, Cornell University, "Real-time Control of Ambulance Services"

Sally McClean, University of Ulster, Londonderry, "Integrating Methods and Practice Across Health, Social and Community Services"

Haipeng Shen, University of North Carolina, Chapel Hill, "Statistical Modeling of Labor-Intensive Service Systems"

Young-Jun Son, University of Arizona, "A Multi-Scale Crowd Behavior Modeling Framework: Emergency Evacuation and Normal Scenarios"

Huseyin Topaloglu, Cornell University, "Revenue Management: Applications, Models and Algorithms"

Assaf Zeevi, Columbia University, "Model Misspecification and Sequential Stochastic Optimization"

The program, which includes the abstracts of all plenary and poster presentations, is available at https://symposia.gerad.ca/2011-ISIM-ResearchWorkshop/en.

Several key themes weaved throughout the events of the workshop, which may prove useful in informing and guiding simulation research on complex service systems. These can be broken down into modeling and methodological themes.

(c) 2014 ACM 1049-3301/2014/01-ART1 $\$ 15.00$

DOI: http://dx.doi.org/10.1145/2555690 


\section{Modeling}

- Models that are composites of smaller models will grow in importance and use.

- It is essential to appropriately capture human behavior in service-system models.

- Service systems may require great care in modeling inputs and the service process.

- Visualization and human-in-the-loop modeling are important growth areas.

- As with all modeling, one must take care to capture an appropriate level of detail: not too much.

\section{Methodology}

- More work is needed in understanding input uncertainty and model misspecification.

- Simulation modeling must explicitly consider the robustness of the resulting recommendations.

- There are potentially strong links with approximate dynamic programming for realtime applications.

- Methods for combining deterministic and simulation optimization are needed.

The five articles published in this special issue were selected based on the quality of their contribution and their link to the main themes.

Advanced stochastic optimization techniques are essential for effective combination of traditional and alternative energy sources in order to unlock the potential of emerging smart energy grids. In "Two-Stage Stochastic Optimization for Optimal Power Flow Under Renewable Generation Uncertainty," Dzung Phan and Soumyadip Ghosh propose a two-stage stochastic version of the classical economic dispatch problem, a formulation that is central to power transmission and distribution over an electricity grid. Generation decisions made in the first stage - such as bringing bulk thermal, nuclear, and hydro generators online-cannot further be changed in the second stage, where the uncertainty due to various factors is realized, such as the amount of power generated from wind and solar sources. Discrepancies between supply and demand in the second stage must be alleviated using high marginal-cost power sources that can be tapped in short order. Unlike many prior formulations, the current model explicitly captures Alternating Current (AC) power flow constraints, which leads to a challenging nonconvex optimization problem. The authors solve a two-stage nonconvex Sample-Average Approximation (SAA) of this formulation, capturing the uncertainty using a finite number of scenario samples. They provide two outer approximation algorithms for finding a globally optimal solution and prove convergence by exploiting recently discovered structural properties for the classical deterministic problem. They demonstrate experimentally the advantages of their approach over a more traditional technique based on the alternating direction method of multipliers.

Moving on from power generation, the next article focuses on a major consumer of energy: the ubiquitous and often enormous data centers used by Web companies, utilities, and others. Improvements in energy efficiency can potentially save billions of dollars in energy costs. In "GDCSim-A Tool for Analyzing Green Data Center Design and Resource Management Techniques," Sandeep K. S. Gupta, Ayan Banerjee, Zahra Abbasi, Georgios Varsamopoulos, Michael Jonas, Joshus Ferguson, Rose Robin, Gilbert, and Tridib Mukherjee present a framework and a software tool called the Green Data Center Simulator (GDCSim) for the iterative design and development of energy-efficient data centers. This unified design tool avoids the need for designers to be deeply familiar with a large number of domain-specific design tools that require user intervention in each step of the design process.

The next article presents a powerful and flexible system for complex epidemic simulations based on a novel combination of several high-performance computing technologies. In "INDEMICS: An Interactive High-Performance Computing 
Framework for Data-Intensive Epidemic Modeling," Keith Bisset, Jiangzhuo Chen, Suruchi Deodhar, Xizhou Feng, Yifei Ma, and Madhav Marathe describe the design and prototype implementation of the INDEMICS (Interactive Epidemic Simulation) modeling and simulation environment, whose goal is to help policy analysts and epidemiologists in planning for and controlling pandemics. INDEMICS provides a simple and powerful way to represent and analyze policy-based (e.g., closing schools, providing vaccinations) and individual-based (e.g., self-isolation) interventions. Users can stop the simulation at any point, assess the state of the simulated system, and add additional interventions based on the current system state. The rich expressiveness, ease of use, and high performance of the system derive from a novel technical approach in which a detailed network model of disease spread is executed in a high-performance computing environment while a relational database system is used to query and summarize the state of the disease model for the user and to allow the user to express complex disease control policies in a concise manner. The authors demonstrate the capabilities of the system through some real case studies.

Trace-driven simulations of complex systems have been in wide use for a long time. Although the use of trace inputs avoids the need to impose (possibly oversimplified) distributional assumptions, the simulation results do not generalize well to other scenarios, thus, it is hard to analyze system variability and hence risk. Lee W. Schruben and Dashi I. Singham address this issue in their article, "Data-Driven Simulation of Complex Multidimensional Time Series," by introducing a framework for resampling general time series data. This resampling method, inspired by computer agent flocking algorithms, can be used not only to generate inputs to complex simulation models but also to generate pseudoreplications of expensive simulation outputs. The authors show how their technique can be applied to emergency department data.

Stochastic Approximation (SA) is widely known as a general and powerful method to optimize continuous parameters in complex stochastic systems. However, the SA algorithm is very sensitive to the selection of its parameters (e.g., the tuning sequence), and a poor selection can reduce the convergence rate. A good parameter selection must depend on the (unknown) function to be optimized and is very difficult to make in general, in particular when this function is high dimensional. In "Multidimensional Stochastic Approximation: Adaptive Algorithms and Applications," Mark Broadie, Deniz M. Cicek, and Assaf Zeevi propose adaptive versions of SA algorithms that dynamically scale and shift the tuning sequences to better match the characteristics of the underlying function and the noise level. They demonstrate good empirical performance of their algorithms on a wide range of examples that include inventory management, health care, revenue management, supply chain management, financial engineering, and queueing.

The workshop organizers, co-chairs, and Special Issue guest editors:

Peter J. Haas, IBM Research Shane G. Henderson, Cornell University Pierre L'Ecuyer, University of Montreal November 26, 2013 